\title{
Intracellular lysozyme and lactoferrin in myeloproliferative disorders
}

\author{
D. Y. MASON
}

From the Department of Haematology, Gibson Laboratories, Radcliffe Infirmary, Oxford

SUMMARY Samples from 49 cases of myeloproliferative diseases were tested by an immunocytochemical technique for leucocyte lysozyme and lactoferrin. The presence of these constituents in myeloid precursors from cases of acute and chronic myeloid leukaemia reflected the degree of cellular maturation, lysozyme appearing (as it does in normal myeloid cells) at the stage of primary granule production (in promyelocytes), while lactoferrin was detectable only in more mature, secondary granule-containing myeloid cells. Auer rods stained positively for lysozyme, in keeping with their relationship to primary granules.

Monocytes from five cases of leukaemia showing predominantly monocytic differentiation were indistinguishable from normal monocytes in their staining reactions for lysozyme despite the presence of raised serum and urinary lysozyme levels.

In four cases of acute myeloid leukaemia circulating polymorphs deficient in lactoferrin were detected: in one of these cases a similar percentage of polymorphs was lysozyme negative.

In a recent report from this laboratory an immunocytochemical technique was described for the demonstration of cytoplasmic lysozyme and lactoferrin in human white cells (Mason et al., 1975). The first of these constituents is present in both myeloid and monocytic cells; the second is restricted to myeloid cells.

The present report is concerned with the distribution of these two substances in leucocytes from cases of myeloproliferative diseases. This study was designed, first, to investigate whether the demonstration of lysozyme and lactoferrin might aid the identification or classification of proliferating cells in these disorders; secondly, to study whether cases of monocytic leukaemia associated with marked increases in blood and urinary lysozyme are characterised by abnormally strong reactions for intracellular lysozyme: and, finally, to look for evidence of acquired deficiencies of intracellular lysozyme or lactoferrin in myeloid leukaemia and related diseases.

\section{Patients and methods}

PATIENTS

Samples were obtained from patients attending the

Received for publication 28 October 1976
Radcliffe Infirmary, Oxford. The diagnosis of leukaemia and of other myeloproliferative disorders was based upon standard clinical and laboratory criteria. The number of patients in each diagnostic category is given in Table 1. It should be noted that three cases of primary sideroblastic anaemia and paroxysmal nocturnal haemoglobinuria ( $\mathrm{PNH}$ ) have been included (under the category of chronic myeloproliferative disorders). Although not conventionally

Table 1 Patients tested by the immunocytochemical technique for intracellular lysozyme and lactoferrin

\begin{tabular}{lc}
\hline Diagnosis & $\begin{array}{l}\text { No. of } \\
\text { patients }\end{array}$ \\
\hline Chronic myeloproliferative disorders & \\
1 Chronic myeloid leukaemia & 14 \\
2 Myelofibrosis & 6 \\
3 Polycythaemia rubra vera & 7 \\
4 Primary sideroblastic anaemia & 2 \\
5 Paroxysmal nocturnal haemoglobinuria & 1 \\
& \\
Acute/subacute myeloproliferative disorders & 14 \\
6 Acute myeloblastic/myelomonocytic/promyelocytic & 2 \\
leukaemia & 3 \\
8 Subacute myelomonocytic leukaemia & \\
\hline
\end{tabular}

All cases were tested for both lysozyme and lactoferrin reactivity, with the exception of two patients in group 6 and one in group 7 who were tested for lysozyme only. 
classified in this way their association with acute leukaemia, and the neutrophil alkaline phosphatase deficiency which characterises PNH justifies their inclusion.

All cases of acute leukaemia were studied before the initiation of treatment, whereas the majority of cases of chronic myeloproliferative diseases were investigated while under treatment. However, all patients in this category had evidence of active disease at the time of testing.

\section{SAMPLES}

Smears of whole blood or buffy coat preparations were made from EDTA anticoagulated venous samples within four hours of specimen collection. These smears, and direct smears of marrow aspirates, were stored at room temperature for a maximum of five days before being stained. Previous studies had established that no loss of lysozyme or lactoferrin reactivity occurs during this period.

\section{IMMUNOPEROXIDASE STAINING OF LYSOZYME} AND LACTOFERRIN

The technique used for the demonstration of these constituents has been described previously and was applied without modification (Mason et al., 1975). In summary, the method involves the successive application to fixed cell smears (after inhibition of endogenous peroxidase) of: (1) specific rabbit antiserum to lysozyme or lactoferrin; (2) swine antiserum to rabbit immunoglobulin; and (3) soluble complexes of horseradish peroxidase and rabbit antibody to this enzyme. The swine antiserum (2) acts as a 'bridge' between the primary antibody and the peroxidase-containing complexes. The development of the peroxidase activity by a cytochemical technique (using diaminobenzidine and hydrogen peroxide as substrates) allows identification of lysozyme or lactoferrin containing cells. Slides were counterstained with haematoxylin and mounted in DPX.

Results

NORMALS

The immunocytochemical reactions of normal peri- pheral blood and bone marrow samples for lysozyme and lactoferrin have been reported previously (Mason et al., 1975) and are summarised in Table 2.

\section{CHRONIC MYELOPROLIFERATIVE DISORDERS}

\section{Chronic myeloid leukaemia}

All 14 patients in this category gave normal staining reactions for the two antigens in mature neutrophils and monocytes. Neutrophil precursors showed a reaction consistent with their stage of maturation, ie myeloblasts were negative for both antigens, promyelocytes stained for lysozyme only, while both constituents were detected in metamyelocytes and band forms.

In two cases of chronic myeloid leukaemia circulating polymorphs were observed lacking each antigen. However, both these cases were characterised by increased basophil counts, and the percentage of lysozyme and lactoferrin deficient polymorphs correlated with the basophil count.

Polycythaemia rubra vera, myelofibrosis, paroxysmal nocturnal haemoglobinuria, and sideroblastic anaemia In each of the 14 cases in this category normal neutrophil and monocyte staining reactions were obtained for both antigens.

\section{ACUTE MYELOPROLIFERATIVE DISORDERS}

\section{Acute myeloblastic/myelomonocytic leukaemia}

Myeloid precursors in the 14 cases in this group showed staining reactions for lysozyme and lactoferrin which paralleled their degree of cellular maturation as assessed on morphological grounds and from Sudan Black staining. The percentage of lysozyme positive precursors was consistently greater than that of lactoferrin positive cells.

The highest percentage of lysozyme positive leukaemic marrow cells was observed in a case of acute promyelocytic leukaemia, in keeping with the large number of cells containing azurophil granules. In one case of acute myeloblastic leukaemia Auer rods were plentiful. These structures stained positively for lysozyme but not for lactoferrin. The lysozyme reaction in most cells in this case was

Table 2 Summary of immunoperoxidase reactions for lysozyme and lactoferrin in different classes of human hite cells

\begin{tabular}{|c|c|c|c|c|c|c|c|c|}
\hline & \multicolumn{5}{|c|}{ Neutrophil myeloid } & \multirow{2}{*}{$\frac{\text { Monocytic }}{\text { Monocytes }}$} & \multirow{2}{*}{$\begin{array}{l}\text { Erythroid } \\
\text { Normoblasts } \\
\text { and red cells }\end{array}$} & \multirow{2}{*}{$\frac{\text { Lymphoid }}{\text { Lymphocytes }}$} \\
\hline & M'blasts & Prom'cytes & Myelocytes & Metam'cytes & $\begin{array}{l}\text { Band and } \\
\text { segmented forms }\end{array}$ & & & \\
\hline Lysozyme & - & + & + & + & + & \pm & - & - \\
\hline Lactoferrin & - & - & + & + & + & $\overline{-}$ & - & - \\
\hline
\end{tabular}

- absent; + present; \pm present in some cells, absent in others. 
Table 3 Details of leukaemic patients in whom lysozyme and/or lactoferrin deficient neutrophils were detected

\begin{tabular}{lll}
\hline Diagnosis & $\begin{array}{l}\text { Lysozvme negative } \\
\text { neutrophils } \\
\%\end{array}$ & $\begin{array}{l}\text { Lactoferrin negative } \\
\text { neutrophils } \\
\%\end{array}$ \\
\hline $\begin{array}{l}\text { Acute myelomonocytic } \\
\text { leukaemia }\end{array}$ & $<5$ & 36 \\
$\begin{array}{c}\text { Acute myelomonocytic } \\
\text { leukaemia }\end{array}$ & $<5$ & 60 \\
$\begin{array}{c}\text { Subacute } \\
\text { myelomonocytic } \\
\text { leukaemia }\end{array}$ & 29 & 30 \\
$\begin{array}{c}\text { Subacute } \\
\text { myelomonocytic } \\
\text { leukaemia }\end{array}$ & $<5$ & 8 \\
\hline
\end{tabular}

confined to the margin of the Auer rods, outlining negatively staining central clefts.

Circulating polymorphs showed normal staining for both antigens (more than $95 \%$ positive) in 12 of the 14 cases of acute myeloblastic and myelomonocytic leukaemia studied. In the remaining two patients (Table 3 ) a population of negatively staining peripheral blood neutrophils was identified. The Figure illustrates one of these cases, $36 \%$ of whose peripheral polymorphs were deficient in lactoferrin. Careful examination of the Romanovsky stained peripheral film from this patient did not reveal any agranular or otherwise atypical polymorphs.

Circulating monocytes from all 14 patients stained normally for lysozyme. In cases where there was a mixed peripheral population of monocytoid and myelocytic cells (ie, acute 'myelomonocytic' leukaemia) lysozyme staining clearly differentiated between the weak to negatively staining monocytoid population and the intensely staining immature myeloid cells.

\section{ACUTE MONOBLASTIC/MONOCYTIC}

LEUKA EMIA

Two patients were studied whose leukaemic cells showed predominantly monocytic differentiation, associated with greatly raised serum and urinary lysozyme levels. In both cases the staining pattern of the leukaemic monocytes was very similar to that of normal monocytes, ie, a weak or negative stain for lysozyme and a negative reaction for lactoferrin. No monocytoid cells stained for lysozyme with an intensity comparable to that consistently seen for myeloid cells. Faint lysozyme positive 'halos' were not infrequently observed around leukaemic monocytes, suggesting that some lysozyme had escaped from these cells as the smears were prepared.

SUBACUTE/CHRONIC MYELOMONOCYTIC

LEUKAEMIA

The three patients in this category conformed to the clinicopathological pattern described by Sexauer $e t$ al. (1974) and Geary et al. (1975) in that they were elderly patients with a peripheral monocytosis, myelomonocytic bone marrow infiltration, and increased serum lysozyme levels.

The monocytoid cells from these patients showed weak staining reactions for lysozyme resembling normal monocytoid cells; as in the case of acute monocytic leukaemia (see section above), no monocytes stained as strongly for lysozyme as did myeloid cells. Monocytes from these patients were lactoferrin negative.

In two of these patients lactoferrin negative mature polymorphs were detected, associated in one case with a similar percentage of negative polymorphs in the preparation stained for lysozyme (Table 3 ).

\section{Discussion}

One of the aims of this study was to assess whether staining for lactoferrin and lysozyme may be of value in the classification or identification of leukaemic cells. The results obtained suggest that these reactions yield no more information than can be obtained from conventional techniques such as the assessment of cell morphology and the use of cytochemical stains. Lysozyme positivity appeared to parallel sudanophilia (and hence, presumably, peroxidase activity) in leukaemic precursors. Furthermore, the reaction of Auer rods for lysozyme resembled that previously described for myeloperoxidase.

This is in keeping with the fact that myeloperoxidase and lysozyme appear simultaneously in the course of normal myeloid maturation (at the promyelocyte stage), being both primary granule constituents (Spitznagel et al., 1974; Baggiolini et al., 1974; Bainton, 1975a).

Leukaemic myeloblasts (which lack primary granules) were lysozyme negative, a finding which accords with the report by Asamer et al. (1971) that lysozyme could not be visualised by immunofluorescence in leukaemic myeloblasts, although it conflicts with the observation of Karle et al. (1974) that lysozyme can be extracted from leukaemic myeloblasts.

Lactoferrin, as would be expected from its association with secondary granules in normal human myeloid cells (Baggiolini et al., 1974; Spitznagel et al., 1974; Bainton, 1975a) was detected only in more mature leukaemic myeloid precursors. The lactoferrin reaction permitted a clear distinction between late myeloid precursors (positive) and monocytoid cells (negative). However, the same differentiation can equally well be achieved by methods such as esterase staining. 


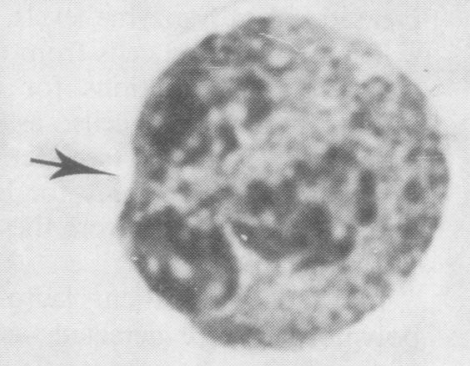

Figure Peripheral blood polymorphs from a case of acute myelomonocytic leukaemia stained for lactoferrin. Note one positive cell (arrowed), characterised by granular cytoplasmic labelling, and an adjacent lactoferrin negative polymorph.

One possible advantage offered by these immunocytochemical techniques is that lactoferrin and lysozyme in blood or marrow smears are relatively stable on storage at room temperature (Mason et al., 1975), so that immunoperoxidase staining may be of value when studying leukaemic samples retrospectively.

A further intention of this investigation was to see whether high levels of serum and urinary lysozyme encountered in cases of leukaemia showing monocytic differentiation would be paralleled by abnormally strong cytochemical reactions for intracellular lysozyme. In the five cases of monocytic leukaemia studied in the present survey, only weak reactions were observed, comparable in their intensity with the reactions of normal monocytes. The report by Perillie et al. (1968) that the intracellular lysozyme content of leukaemic monocytes is less than that of chronic myeloid leukaemic myelocytes and granulocytes accords with this finding. These results pose the paradox that leukaemia cells which are presumably liberating large amounts of lysozyme apparently contain relatively little of this material. One possible explanation is that the im- munocytochemical technique is for some reason inefficient at detecting lysozyme in monocytes. A more probable explanation is that leukaemic monocytes liberate lysozyme rapidly after synthesising it so that no intracellular lysozyme pool accumulates. In contrast, myeloid cells, which store their complement of lysozyme in cytoplasmic granules and liberate it only at cell death or degranulation, react strongly for this protein.

Evidence of continuous liberation of lysozyme from cultured normal human monocytes, and for a greater intracellular content of this material in normal polymorphs than in normal monocytes, has been provided by Gordon et al. (1974). Furthermore, Farhangi and Osserman (1974), studying a case of monocytic leukaemia, were able to demonstrate, after incubation of bone marrow with labelled leucine, that newly synthesised lysozyme accumulated in the medium rather than in the leukaemic cells. Additional evidence for the readiness with which lysozyme is liberated from monocytes is found in their ability to lyse adjacent organisms of Micrococcus lysodeikticus in the cytobacterial technique described by Syrén and Raeste (1971), and in the 'halo' 
effect noted in the present study when staining monocytes for lysozyme by the immunoperoxidase method (see results).

The observation by Asamer et al. (1971) of strong immunofluorescent staining for lysozyme in monocytes from three cases of monocytic leukaemia associated with heavy lysozymuria is in conflict with this concept. This discrepancy is conceivably explained by the presence of strongly staining myeloid cells among the monocytic population in their cases: the greater morphological cell detail visible in immunoperoxidase (as opposed to immunofluorescent) preparations is of value in correctly identifying monocytic and myelocytic cells.

Finally, this study sought evidence of acquired deficiencies of cellular lysozyme and/or lactoferrin in myeloproliferative disorders. The deficiency of neutrophil alkaline phosphatase, which is a feature of chronic myeloid leukaemia, suggested that additional deficiencies of neutrophil constituents might be detected in this disorder by immunoperoxidase staining. In particular, a depression or deficiency of lactoferrin reactivity might be expected on the grounds that alkaline phosphatase has been localised (in common with lactoferrin) to neutrophil secondary granules (Bainton, 1975a).

In the present study no abnormalities of either lactoferrin or lysozyme reactivity were observed in chronic myeloid leukaemic neutrophils. It may be noted that recent work (Bretz and Baggiolini, 1974; Spitznagel et al., 1974) has shown that alkaline phosphatase reactivity is in fact not associated with either primary or secondary human neutrophil granules but with a third subcellular fraction. Furthermore, electron microscopic examination of chronic myeloid leukaemic neutrophils reveals a normal complement of primary and secondary granules (Ullyot and Bainton, 1974) so that normal lactoferrin and lysozyme neutrophil reactivity in this disease is not an inconsistent finding. There has, however, been a recent report from Olofsson et al. (1975) suggesting that in a proportion of chronic myeloid leukaemia patients intracellular levels of neutrophil lysozyme and lactoferrin are reduced.

It is possible that the discrepancy between the findings of Olofsson et al. and those of the present study arises from the fact that immunocytochemical methods are only semiquantitative and, while being able to reveal even a small subpopulation of grossly deficient neutrophils, cannot detect a generalised reduction in lysozyme or lactoferrin content affecting the majority of cells.

In contrast to the findings in chronic myeloid leukaemia and other chronic myeloproliferative disorders, it was possible to demonstrate lysozyme and lactoferrin deficient neutrophils in a proportion of cases of acute myeloid leukaemia. This is the first occasion on which deficiencies of these two white cell constituents have been directly demonstrated in individual cells, although Olsson's group (Odeberg et al., 1976) has recently reported a marked reduction in the quantity of lactoferrin extractable from neutrophils in cases of acute myeloid leukaemia, and a similar observation was made by Karle et al. (1974) for neutrophil lysozyme in this disease. Furthermore, Bainton (1975b) has reported deficiencies of both primary and secondary granules on ultrastructural examination of granulocytes from cases of acute myeloid leukaemia.

A close precedent to this leukaemia-associated deficiency of lysozyme and lactoferrin is provided by the deficiency of neutrophil myeloperoxidase which has been reported by a number of authors in cases of acute leukaemia (Hayhoe et al., 1967; Schmalzl et al., 1970; Davis et al., 1971; Catovsky et al., 1972), presumably representing the production of abnormal polymorphs by the leukaemic myeloid clone. One point of difference from leukaemia-associated myeloperoxidase deficiency is found in the fact that lysozyme and lactoferrin deficiency was found in the present study in association with both myeloblastic leukaemia, whereas Catovsky et al. (1972) emphasised that neutrophil myeloperoxidase deficiency was rare in cases showing predominantly monocytic differentiation.

It remains to be determined whether deficiency of neutrophil lysozyme or lactoferrin can be incriminated as a cause of infectious complications in acute leukaemia. One obvious practical implication of these neutrophil deficiencies is that their demonstration may provide additional diagnostic information in the study of atypical myeloproliferative states such as 'preleukaemia' or refractory anaemia since it has already been demonstrated that myeloperoxidase deficiency can occur in these disorders (Catovsky et al., 1971; Bessis et al., 1969). Future studies could profitably explore this avenue and also attempt to correlate, in individual cases of leukaemia, quantitative assays of granular proteins extracted from leukaemic cells with the pattern of immunocytochemical reactions for the same constituents.

I am grateful to Caroline Farrell for skilful technical assistance; to the Haematology Department and Nuffield Department of Medicine, Radcliffe Infirmary for samples from patients under their care; and to Dr T. Parry for photographic assistance.

\section{References}

Asamer, H., Schmalzl, F., and Braunsteiner, H. (1971). Immunocytological demonstration of lysozyme (mura- 
midase) in human leukaemic cells. British Journal of Haematology, 20, 571-574.

Baggiolini, M., Bretz, U., and Gusus, B. (1974). Biochemical characterization of azurophil and specific granules from human and rabbit polymorphonuclear leukocytes. Schweizerische medizinische Wochenschrift, 104, 129-132.

Bainton, D. F. (1975a). Neutrophil granules. British Journal of Haematology, 29, 17-21.

Bainton, D. F. (1975b). Abnormal neutrophils in acute myelogenous leukemia: identification of subpopulations based on analysis of azurophil and specific granules. Blood Cells, 1, 191-199.

Bessis, M., Dreyfus, B., Breton-Gorius, J., and Sultan, C. (1969). Étude au microscope électronique de onze cas d'anémies réfractaires avec enzymopathies multiples. Nouvelle Revue Française d'Hématologie, 9, 87104.

Bretz, U. and Baggiolini, M. (1974). Biochemical and morphological characterisation of azurophil and specific granules of human neutrophilic polymorphonuclear leukocytes. Journal of Cell Biology, 63, 251-269.

Catovsky, D., Shaw, M. T., Hoffbrand, A. V., and Dacie, J. V. (1971). Sideroblastic anaemia and its association with leukaemia and myelomatosis: a report of five cases. British Journal of Haematology, 20, 385-393.

Catovsky, D., Galton, D. A. G., and Robinson, J. (1972). Myeloperoxidase-deficient neutrophils in acute myeloid leukaemia. Scandinavian Journal of Haematology, 9, 142-148.

Davis, A. T., Brunning, R. D., and Quie, P. G. (1971). Polymorphonuclear leucocyte myeloperoxidase deficiency in a patient with myelomonocytic leukaemia. New England Journal of Medicine, 285, 789-790.

Farhangi, M. and Osserman, E. F. (1974). De novo synthesis of lysozyme by bone marrow cells of a patient with monomyelocytic leukemia. In Lysozyme, edited by E. F. Osserman, R. E. Canfield, and S. Beychok, pp. 379-383. Academic Press, London.

Geary, C. G., Catovsky, D., Wiltshaw, E., Milner, G. R., Scholes, M. C., Van Noorden, S., Wadsworth, L. D., Muldal, S., MacIver, J. E., and Galton, D. A. G. (1975). Chronic myelomonocytic leukaemia. British Journal of Haematology, 30, 289-302.
Gordon, S., Todd, J., and Cohn, Z. A. (1974). In vitro synthesis and secretion of lysozyme by mononuclear phagocytes. Journal of Experimental Medicine, 139, 1228-1248.

Hayhoe, F. G. J., Quaglino, D., and Doll, R. (1967). The Cytology and Cytochemistry of Acute Leukaemias. HMSO, London.

Karle, H., Hansen, N. E., and Killmann, S. A. (1974). Intracellular lysozyme in mature neutrophils and blast cells in acute leukemia. Blood, 44, 247-255.

Mason, D. Y., Farrell, C., and Taylor, C. R. (1975). The detection of intracellular antigens in human leucocytes by immunoperoxidase staining. British Journal of Haematology, 31, 361-390.

Odeberg, H., Olofsson, T., and Olsson, I. (1976). Primary and secondary granule contents and bactericidal capability of neutrophils in acute leukaemia. Blood Cells (In press).

Olofsson, T., Odeberg, H., and Olsson, I. (1975). Granulocyte function in chronic granulocytic leukaemia. Bactericidal and metabolic capabilities. Paper presented at International Society of Haematology Meeting, London, August 1975.

Perillie, P. E., Kaplan, S. S., Lefkowitz, E., Rogaway, W., and Finch, S. C. (1968). Studies of muramidase (lysozyme) in leukemia. Journal of the American Medical Association, 203, 317-322.

Schmalzl, W., Lederer, B., and Braunsteiner, H. (1970). Atypical myeloblastic leukaemia with differentiation into "paraneutrophils". Blut, 20, 337-349.

Sexauer, J., Kass, L., and Schnitzer, B. (1974). Subacute myelomonocytic leukaemia. American Journal of Medicine, 57, 853-861.

Spitznagel, J. K., Dalldorf, F. G., Leffell, M. G., Folds, J. D., Welsh, I. R. H., Cooney, M. H., and Martin, L. E. (1974). Character of azurophil and specific granules purified from human polymorphonuclear leukocytes. Laboratory Investigation, 30, 774-785.

Syrén, E. and Raeste, A-M. (1971). Identification of blood monocytes by demonstration of lysozyme and peroxidase activity. Acta Haematologica, 45, 29-35.

Ullyot, J. L. and Bainton, D. F. (1974). Azurophil and specific granules of blood neutrophils in chronic myelogenous leukemia: an ultrastructural and cytochemical analysis. Blood, 44, 469-482. 